Article

\title{
Equations of State for Hadronic Matter and Mass-Radius Relations of Neutron Stars with Strong Magnetic Fields
}

\author{
Chinatsu Watanabe *(D), Naotaka Yoshinaga $(\mathbb{D}$ and Shuichiro Ebata $\mathbb{D}$ \\ Department of Physics, Saitama University, Saitama 338-8570, Japan; yoshinaga@phy.saitama-u.ac.jp (N.Y.); \\ ebata@mail.saitama-u.ac.jp (S.E.) \\ * Correspondence: watanabe@gen.th.phy.saitama-u.ac.jp
}

\begin{abstract}
Neutron star is an important object for us to verify the equation of state of hadronic matter. For a specific choice of equations of state, mass and radius of a neutron star are determined, for which there are constraints from observations. According to some previous studies, since the strong magnetic field acts as a repulsive force, there is a possibility that neutron stars with strong magnetic fields may have relatively heavier masses than other non-magnetized neutron stars. In this paper, the structure of a neutron star with a strong internal magnetic field is investigated by changing its internal functional form to see how much the neutron star can be massive and also how radius of a neutron star can be within a certain range.
\end{abstract}

Keywords: nuclear matter; magnetar; hadronic EoS

check for updates

Citation: Watanabe, C.; Yoshinaga, N.; Ebata, S. Equations of State for Hadronic Matter and Mass-Radius Relations of Neutron Stars with Strong Magnetic Fields. Universe 2022, 8, 48. https://doi.org/ 10.3390 /universe 8010048

Academic Editors: David Blaschke, Konstantin Maslov, Elena Litvinova and Evgeni Kolomeitsev

Received: 27 December 2021 Accepted: 6 January 2022 Published: 12 January 2022

Publisher's Note: MDPI stays neutral with regard to jurisdictional claims in published maps and institutional affiliations.

Copyright: (C) 2022 by the authors Licensee MDPI, Basel, Switzerland. This article is an open access article distributed under the terms and conditions of the Creative Commons Attribution (CC BY) license (https:// creativecommons.org/licenses/by/ $4.0 /)$

\section{Introduction}

To study a neutron star (NS) is an interesting and important subject in nuclear physics as it is a unique object of superdense hadronic matter, where its central density might be several times larger than the nuclear saturation density. Equation of state (EoS) for the nuclear matter that depends on the energy density functional is often utilized to discuss various properties of NSs, for instance, the mass-radius (MR) relation.

Historically, Oppenheimer and Volkoff (independently, Tolman) were the first who estimated its maximum mass of $M_{\max } \sim 0.71$ solar mass $\left(M_{\odot}\right)$ by employing a noninteracting strongly degenerate relativistic gas of neutrons in 1939 [1,2]. Discoveries of NSs with masses around twice the solar mass have made a strong impact on the nuclear physics community who had once believed that the maximum mass of an NS should not exceed $1.56 M_{\odot}$ due to the occurrence of kaon condensation [3,4]. Indeed, several NSs with around $2 M_{\odot}$ have been confirmed since 2010 . The NS PSR J1614-2230 has the observed mass of $1.97 \pm 0.04 M_{\odot}$ [5] and the NS PSR J0348+0432 has mass of $2.01 \pm 0.04 M_{\odot}$ [6]. In 2021, the mass and equatorial radius of the millisecond-pulsar (MSP) J0740+6620 was constrained to be $M=2.072_{-0.066}^{+0.067} M_{\odot}$ and $R_{2.072 M_{\odot}}=12.39_{-0.98}^{+1.30} \mathrm{~km}$, respectively [7-10]. If NS matter consists of only nucleons with strong two- or three-body repulsive forces, massive NSs more than $2 M_{\odot}$ can be easily described. However, hyperons are energetically favorable when the baryon chemical potential is large enough in the inner core of an NS. This leads to a reduction in the Fermi pressure to soften the EoS and to a reduction in the predicted maximum mass less than $2 M_{\odot}$. This is called the hyperon puzzle. Since discoveries of massive NSs, many people have searched for the optimal EoSs that can achieve masses over $2 M_{\odot}$. To obtain NS masses more than $2 M_{\odot}$ is one of the goals to be achieved for the optimal EoSs.

There are some characteristic NSs: millisecond-pulsar, which has a short rotational period of less than $10 \mathrm{~ms}$, and magnetar, which has a powerful magnetic field on its surface. As a part of external conditions to support over $2 M_{\odot}$, the extremely rapid rotation and powerful magnetic field should be considered in the EoS study. In our recent studies [11,12], EoSs for a rapidly rotating NS with some deformation and a fixed magnetic field have 
been investigated. Although an unrealistically rapid rotation can modify the NS mass from $1.89 M_{\odot}$ to $2 M_{\odot}$, the rotational effects on the mass increase were found to be not so significant for the realistic frequency range of the observed millisecond-pulsars.

A magnetar is a type of the NS that has a strong magnetic field on the surface. Recently, up to 30 magnetars have been observed $[13,14]$. Maximumly, about $10^{15} \mathrm{G}$ of the magnetic field on the surface has been found by the observation [13], but we do not yet know the generation mechanism of the strong magnetic fields, where and how they come from.

Another constraint on the EoS comes from the NS radius. From the observation of the GW170817 gravitational wave event, the upper limit radius is $13.6 \mathrm{~km}$ [15] at the mass of $M=1.4 M_{\odot}$. Moreover, another reports the upper limit radius $13.76 \mathrm{~km} \mathrm{[16]} \mathrm{at} M=1.4 M_{\odot}$. The lower limit radius was reported as $10.68_{-0.04}^{+0.15} \mathrm{~km}$ [17] at $M=1.6 M_{\odot}$. The upper limit of radius by NICER observations of PSR J0030+0451 is $13.02_{-1.06}^{+1.24} \mathrm{~km}$ [18] at $M=1.44_{-0.14}^{+0.15} M_{\odot}$.

In this paper, the internal structure and MR relation of NS, including hyperon populations, are investigated by changing the functional form of a strong magnetic field. The strength of the magnetic field is fixed constant as $10^{12} \mathrm{G}$ on the surface and over $10^{18} \mathrm{G}$ near the center. The shape of the magnetic field is parametrized by two parameters $\alpha$ and $\gamma$. We first formulate the relativistic mean field (RMF) theory, including hyperons. Here, we introduce 12 different EoSs $[19,20]$ depending on the different coupling constants between baryons and mesons. These EoSs have similar saturation properties at nuclear saturation density. This RMF includes a strong magnetic field, which has a functional form of baryon number density $\rho$. In this work, we solve the Tolman-Oppenheimer-Volkov (TOV) equations to obtain masses and radii for various EoSs by changing $\alpha$ and $\gamma$ parameters freely. Here, the MR relations are verified with respect to the constraints that maximum masses are within the range of observed masses and the radii at 1.4 solar mass are less than $13.76 \mathrm{~km}$.

This paper is organized as follows. The formulation of the calculation is given in Section 2. The results are given in Section 3. Finally, a summary is given in Section 4.

\section{Formulations}

\subsection{Equation of State}

We adopt the relativistic mean field (RMF) theory based on the nonlinear Walecka model in this work. We assume that the NS matter is static and uniform in the high density region. The Lagrangian reads [19-26]

$$
\mathcal{L}=\sum_{b} \mathcal{L}_{b}+\mathcal{L}_{m}+\sum_{l} \mathcal{L}_{l}+\mathcal{L}_{e m}
$$

where

$$
\begin{aligned}
\mathcal{L}_{b}= & \bar{\psi}_{b}\left(i \gamma_{\mu} \partial^{\mu}-m_{b}+g_{\sigma b} \sigma+g_{\sigma^{*} b} \sigma^{*}-g_{\omega b} \gamma_{\mu} \omega^{\mu}\right. \\
& -g_{\phi b} \gamma_{\mu} \phi^{\mu}-g_{\rho b} \gamma_{\mu} \boldsymbol{\tau} \cdot \boldsymbol{\rho}^{\mu} \\
& \left.-q_{b} \gamma_{\mu} A^{\mu}-\kappa_{b} \sigma_{\mu \nu} F^{\mu v}\right) \psi_{b}, \\
\mathcal{L}_{m}= & \frac{1}{2}\left(\partial_{\mu} \sigma \partial^{\mu} \sigma-m_{\sigma}^{2} \sigma^{2}\right)+\frac{1}{2}\left(\partial_{\mu} \sigma^{*} \partial^{\mu} \sigma^{*}-m_{\sigma^{*}}^{2} \sigma^{* 2}\right) \\
+ & \frac{1}{2} m_{\omega}^{2} \omega_{\mu} \omega^{\mu}-\frac{1}{4} \Omega_{\mu \nu} \Omega^{\mu v}+\frac{1}{2} m_{\phi}^{2} \phi_{\mu} \phi^{\mu} \\
- & \frac{1}{4} \Phi_{\mu \nu} \Phi^{\mu v}+\frac{1}{2} m_{\rho}^{2} \boldsymbol{\rho}_{\mu} \cdot \boldsymbol{\rho}^{\mu}-\frac{1}{4} \boldsymbol{P}^{\mu v} \cdot \boldsymbol{P}_{\mu v} \\
- & \frac{1}{3} b m_{n}\left(g_{\sigma} \sigma\right)^{3}-\frac{1}{4} c\left(g_{\sigma} \sigma\right)^{4} \\
+ & \frac{1}{4 !} \xi\left(g_{\omega}^{2} \omega_{\mu} \omega^{\mu}\right)^{2}+\Lambda_{\omega}\left(g_{\omega}^{2} \omega_{\mu} \omega^{\mu}\right)\left(g_{\rho}^{2} \boldsymbol{\rho}_{\mu} \cdot \boldsymbol{\rho}^{\mu}\right),
\end{aligned}
$$




$$
\begin{aligned}
\mathcal{L}_{l} & =\bar{\psi}_{l}\left(i \gamma_{\mu} \partial^{\mu}-q_{l} \gamma_{\mu} A^{\mu}-m_{l}\right) \psi_{l}, \\
\mathcal{L}_{e m} & =-\frac{1}{4} F^{\mu v} F_{\mu v} .
\end{aligned}
$$

Here, $b, m, l$, and em indicate baryons, mesons, leptons, and photons, respectively. The field strengths are explicitly given as

$$
\begin{aligned}
F_{\mu v} & =\partial_{\mu} A_{v}-\partial_{\nu} A_{\mu}, \\
\Omega_{\mu v} & =\partial_{\mu} \omega_{v}-\partial_{\nu} \omega_{\mu}, \\
\Phi_{\mu v} & =\partial_{\mu} \phi_{v}-\partial_{\nu} \phi_{\mu}, \\
\boldsymbol{P}_{\mu v} & =\partial_{\mu} \boldsymbol{\rho}_{v}-\partial_{\nu} \boldsymbol{\rho}_{\mu}-g_{\rho} \boldsymbol{\rho}_{\mu} \times \boldsymbol{\rho}_{\nu} .
\end{aligned}
$$

Here, $\tau / 2$ represents the isospin operator and $\sigma_{\mu v}=\frac{i}{2}\left[\gamma_{\mu}, \gamma_{v}\right]$, where $\gamma_{\mu}$ is Dirac $\gamma$ matrix. Characters in bold font represent isovector fields. The baryon octet $p, n, \Lambda, \Sigma^{0}, \Sigma^{ \pm}$, $\Xi^{0}, \Xi^{-}$, the electron $(0.511 \mathrm{MeV})$, the muon $(105.7 \mathrm{MeV})$, and the tauon $(1777 \mathrm{MeV})$ are considered for fermions [27]. The scalar-meson $\sigma$, the vector-meson $\omega$, and the vector-isovectormeson $\rho$ with masses of $m_{\sigma}=511.198 \mathrm{MeV}, m_{\omega}=783.0 \mathrm{MeV}$, and $m_{\rho}=770.0 \mathrm{MeV}$ are considered for mesons, respectively. In addition, hidden-strangeness mesons, $\sigma^{*}$ and $\phi$ with masses of $m_{\sigma^{*}}=975 \mathrm{MeV}, m_{\phi}=1020 \mathrm{MeV}$ are introduced. The coupling constants of nucleons with these mesons, $g_{\sigma N}, g_{\omega N}$, and $g_{\rho N}$, and some self-interactions among mesons are determined by fitting the physical quantities at the saturation density $[19,21,22]$.

For seven kinds of EoSs employed in the present study, nuclear properties at saturation number density $\rho_{0}$ are given in Table 1 . Here, TM1 and TM2 are different with respect to the slope parameter $L$, where $L$ is closely related to the radius of an NS. The NL3 parametrization is fitted to the ground-state properties of both stable and unstable nuclei. This parametrization predicts very large, purely nucleonic NS maximum masses, but a symmetry energy slope parameter $L$ is too large. Thus, we also consider the parametrization NL3 $\omega \rho$ with a softer density dependence of the symmetry energy due to the inclusion of the nonlinear $\omega \rho$ term.

The parameterizations of twelve EoSs (GM1, GM3, TM1-a, TM1-b, TM2 $\omega \rho-a$, TM2 $\omega \rho-b$, NL3-a, NL3-b, NL3 $\omega \rho-a$, NL3 $\omega \rho-b$, DDME2-a, and DDME2-b) are given as follows. The coupling constants of hyperons with mesons are determined by fitting the properties of hypernuclei in the quark model [20]. In the GM1 parameter set, coupling ratios, $R_{\sigma h}=g_{\sigma h} / g_{\sigma N}=0.6, R_{\omega h}=g_{\omega h} / g_{\omega N}=0.653$, and $R_{\rho h}=g_{\rho h} / g_{\rho N}=0.6$ are adopted. In the GM3 parameter set, coupling ratios, $R_{\sigma h}=g_{\sigma h} / g_{\sigma N}=0.6, R_{\omega h}=g_{\omega h} / g_{\omega N}=0.568$, and $R_{\rho h}=g_{\rho h} / g_{\rho N}=0.6$ are adopted. The GM3 EoS leads to neutron star with compactness smaller than GM1 EoS. As for other couplings of hyperons with the vector and the vector-isovector mesons, the following $\mathrm{SU}(6)$ values are adopted in the a-parameter sets (TM1-a, TM2 $\omega \rho-a$, NL3-a, NL3 $\omega \rho-a$, and DDME2-a):

$$
\begin{aligned}
& R_{\omega \Lambda}=\frac{2}{3}, \quad R_{\omega \Sigma}=\frac{2}{3}, \quad R_{\omega \Xi}=\frac{1}{3} \\
& R_{\rho \Sigma}=2, \quad R_{\rho \Xi}=1, \\
& R_{\phi \Lambda}=-\frac{\sqrt{2}}{3}, \quad R_{\phi \Sigma}=-\frac{\sqrt{2}}{3}, \quad R_{\phi \Xi}=-\frac{2 \sqrt{2}}{3} .
\end{aligned}
$$

The difference of a- and b-parametrizations are as follows: (a) $\mathrm{SU}(6)$, i.e., $R_{\omega \Lambda}=2 / 3$, (b) $R_{\omega \Lambda}=1$, obtained for $R_{\phi \Lambda}$ equal to its SU(6) value and $R_{\sigma \Lambda}$ and $R_{\sigma^{*} \Lambda}$ are calibrated to hypernuclear data with $\Delta B_{\Lambda \Lambda}=0.5 \mathrm{MeV}$. The values of hyperon potentials in symmetric nuclear matter, $U_{\Lambda}^{N}=-30 \mathrm{MeV}, U_{\Sigma}^{N}=0 \mathrm{MeV}$, and $U_{\Xi}^{N}=-14 \mathrm{MeV}$ [20], are used to determine the value of $R_{\sigma h}$. Thus, ratios of the $\sigma-\Lambda$ coupling, the $\sigma$ - $\Sigma$ coupling, and the $\sigma-\Xi$ coupling in EoS parameter sets except GM1 and GM3, are adopted as in Table 2. 
Table 1. Nuclear properties at saturation number density $\left(\rho_{0}\left(\mathrm{fm}^{-3}\right)\right)$ predicted by seven kinds of EoSs used in this study $[20,28]$ : energy per nucleon $(B / A(\mathrm{MeV}))$, incompressibility $(K(\mathrm{MeV}))$, symmetry energy $(J(\mathrm{MeV}))$, its slope parameter $(L(\mathrm{MeV}))$, and curvature $\left(K_{\text {sym }}(\mathrm{MeV})\right)$ at the saturation point of uniform symmetric nuclear matter [29].

\begin{tabular}{llllllll}
\hline & GM1 & GM3 & TM1 & TM2 $\boldsymbol{\omega} \boldsymbol{\rho}$ & NL3 & NL3 $\boldsymbol{\omega} \boldsymbol{\rho}$ & DDME2 \\
\hline$\rho_{0}$ & 0.153 & 0.153 & 0.146 & 0.146 & 0.148 & 0.148 & 0.152 \\
$B / A$ & -16.3 & -16.3 & -16.3 & -16.4 & -16.30 & -16.30 & -16.14 \\
$K$ & 300 & 240 & 281.2 & 281.7 & 271.8 & 271.8 & 250.9 \\
$J$ & 32.5 & 32.5 & 36.9 & 32.1 & 37.4 & 31.7 & 32.3 \\
$L$ & 93.9 & 89.7 & 111.2 & 54.8 & 118.9 & 55.5 & 51.2 \\
$K_{\text {sym }}$ & 17.9 & -6.5 & 33.8 & -70.5 & 101.6 & -7.6 & -87.1 \\
\hline
\end{tabular}

Here, those EoSs with high incompressibility more than $K=240 \mathrm{MeV}$ are considered. All other details are given in [12].

Table 2. Adopted ratios of $\sigma-\Lambda$ coupling, $\sigma-\Sigma$ coupling, and $\sigma-\Xi$ coupling in EoS parameter sets.

\begin{tabular}{|c|c|c|c|}
\hline EoS & $R_{\sigma \Lambda}$ & $R_{\sigma \Sigma}$ & $\boldsymbol{R}_{\sigma \Xi}$ \\
\hline TM1-a & 0.64 & 0.56 & 0.32 \\
\hline TM1-b & 0.91 & 0.56 & 0.32 \\
\hline $\mathrm{TM} 2 \omega \rho-\mathrm{a}$ & 0.64 & 0.55 & 0.32 \\
\hline $\mathrm{TM} 2 \omega \rho-\mathrm{b}$ & 0.93 & 0.55 & 0.32 \\
\hline NL3-a & 0.67 & 0.59 & 0.33 \\
\hline NL3-b & 0.97 & 0.59 & 0.33 \\
\hline NL3 $3 \rho-\mathrm{a}$ & 0.67 & 0.59 & 0.33 \\
\hline NL3 $\omega \rho-b$ & 0.97 & 0.59 & 0.33 \\
\hline DDME2-a & 0.69 & 0.60 & 0.34 \\
\hline DDME2-b & 0.98 & 0.60 & 0.34 \\
\hline
\end{tabular}

\subsection{Magnetic Fields}

The neutron stars can be massive because of the pressure from magnetic fields. In this work, we investigate an NS with a strong magnetic field inside the NS. From observations, only the strength of the magnetic field on the surface is known and we need to assume the strength and the functional form of the magnetic field inside the NS.

In the following, a density-dependent magnetic field strength is adopted in this study $[26,30]$,

$$
B(\rho)=B_{s}+B_{0}\left[1-\exp \left\{-\alpha\left(\frac{\rho}{\rho_{0}}\right)^{\gamma}\right\}\right],
$$

where $B_{0}$ indicates the strength in a denser region than that of the saturation number density $\rho_{0}\left(0.153 \mathrm{fm}^{-3}\right)$ and $B_{s}$ indicates the strength of magnetic fields on the surface. Here, we adopt $B_{S}=10^{12} \mathrm{G}$.

Since Equation (13) does not satisfy div B $=0$ in general, this form of the magnetic fields must be used with caution as a simple way to implement magnetic fields in an NS. With this problem in mind, however, it should be emphasized that for particles in a very small region of an NS, they feel almost a constant magnetic field, for which div $\mathrm{B}=0$ is practically satisfied. For more realistic magnetic fields, different equations might be used [31].

Even if one assumes the form of Equation (13), one has free parameters $\alpha, \gamma$, and $B_{0}$. Up to now, there is no work of changing $\alpha$ and $\gamma$ parameters freely. In this work, we arbitrarily change $\alpha$ and $\gamma$ to investigate their effects on the radii and masses of NSs. 


\subsection{EoS of Hadronic Matter with Magnetic Fields}

The energy density and the pressure of hadronic matter in the presence of the magnetic fields are given as

$$
\begin{aligned}
& \varepsilon=\varepsilon_{m}+B^{2} / 2, \\
& p=p_{m}+B^{2} / 2,
\end{aligned}
$$

respectively, where $\varepsilon_{m}$ and $p_{m}$ are the energy density and the pressure of hadrons where the contribution $B^{2} / 2$ from the magnetic fields are neglected. However, it should be noted that, without $B^{2} / 2$, the effects of the magnetic fields to $\varepsilon_{m}$ and $p_{m}$ are included through the interaction between the magnetic field and each particle's magnetic moment. All other details are given in Appendix A of Reference [12].

\section{Results}

\subsection{Comparison of the Case without Magnetic Fields}

Figure 1 shows the MR relations of 12 EoSs without assuming any magnetic fields. The orange and light green dashed areas indicate pulsars PSR J0740+6620 and PSR J0030+ 0451, respectively. The thick area shows the one within $68 \%$ credibility and the thinner area shows the one within $90 \%$ credibility. Each EoS curve must go through these areas if they satisfy the observational constraints. The arrow indicates the upper limit of radius for $1.4 M_{\odot}$ from the gravitational wave event GW170817.

Only three EoSs (NL3a, NL3 $\omega \rho-\mathrm{a}$, and DDME2-a) barely satisfy the MR constraints (within $90 \%$ credibility for the maximum mass), but among them, only NL3 $\omega \rho$-a $(K=271.8 \mathrm{MeV})$ satisfies the radius constraint at $1.4 \mathrm{M}_{\odot}$. It should be noted that DDME2 $(K=250.9 \mathrm{MeV})$ is a relatively soft EoS at nuclear saturation density, but it becomes harder in a higher density region.

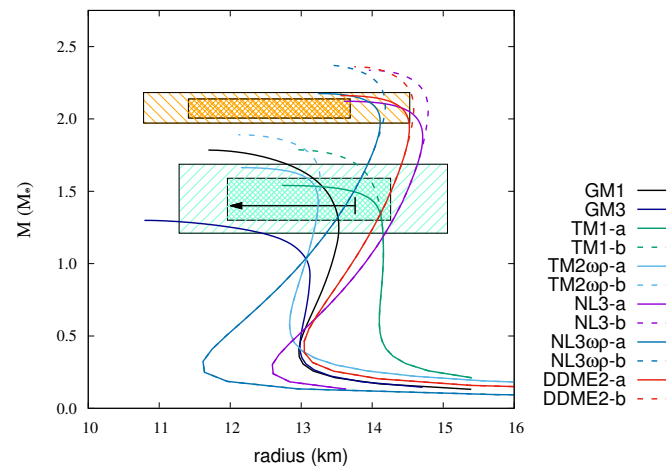

Figure 1. MR relations of 12 EoSs without magnetic fields. The unstable region in each EoS is not shown. The orange and light green dashed areas indicate pulsars PSR J0740+6620 and PSR J0030+0451 (68\% (thick) and 90\% (thin) credibility), respectively. The arrow indicates the upper limit of radius for $1.4 M_{\odot}$ from the gravitational wave event GW170817. The colored lines represent the following: black (solid line), GM1 EoS; green (solid line), TM1-a EoS; green (dashed line), TM1-b EoS; light blue (solid line), TM2 $\omega \rho$-a EoS; light blue (dashed line), TM2 $\omega \rho$-b EoS; purple (solid line), NL3-a EoS; purple (dashed line), NL3-b EoS; dark blue (solid line), NL3 $\omega \rho$-a EoS; dark blue (dashed line), NL3 $\omega \rho$-b EoS; red (solid line), DDME2-a EoS; red (dashed line), DDME2-b EoS.

Table 3 summarizes the maximum masses, radius at $1.4 M_{\odot}$, and radius at $2.072 M_{\odot}$ for 12 EoSs without magnetic fields. Only three kinds of EoSs (NL3, NL3 $\omega \rho$, and DDME2) give masses of twice the solar mass. 
Table 3. Maximum masses, radius at $M=1.4 M_{\odot}$, and radius at $M=2.072 M_{\odot}$ for 12 EoSs without magnetic fields.

\begin{tabular}{llll}
\hline EoS & $\boldsymbol{M}_{\mathbf{m a x}}\left(\boldsymbol{M}_{\odot}\right)$ & $\boldsymbol{R}_{\mathbf{1 . 4}} \boldsymbol{M}_{\odot}(\mathbf{k m})$ & $\boldsymbol{R}_{\mathbf{2 . 0 7 2} \boldsymbol{M}_{\odot}}(\mathbf{k m})$ \\
\hline GM1 & 1.784 & 13.5 & - \\
GM3 & 1.299 & - & - \\
TM1-a & 1.540 & 14.1 & - \\
TM1-b & 1.787 & 14.1 & - \\
TM2 $\omega \rho-\mathrm{a}$ & 1.663 & 13.2 & - \\
TM2 $\omega \rho-\mathrm{b}$ & 1.891 & 13.2 & - \\
NL3-a & 2.122 & 14.4 & 14.4 \\
NL3-b & 2.336 & 14.4 & 14.8 \\
NL3 $\omega \rho-\mathrm{a}$ & 2.175 & 13.6 & 14.1 \\
NL3 $\omega \rho-b$ & 2.372 & 13.6 & 14.2 \\
DDME-a & 2.161 & 14.2 & 14.4 \\
DDME-b & 2.360 & 14.2 & \\
\hline
\end{tabular}

As general features, the maximum mass becomes smaller when hyperons are included, but the maximum mass increases when the magnetic field is implemented. The average radius increases when a strong magnetic field is implemented.

\subsection{Changing $\alpha$ and $\gamma$ Parameters of the Magnetic Field Function}

In this section, the magnetic field $B(\rho)$ in Equation (13) is also implemented, where $\alpha$ and $\gamma$ parameters are changed as free parameters.

The $B(\rho)$ as a function of baryon number density $\rho$ for various $\alpha$ parameters is shown in Figure 2. Each line indicates a magnetic field with $B_{0}=2.5 \times 10^{18} \mathrm{G}$ and $\gamma=2$. In Figure 2, as $\alpha$ becomes larger, the strength of the magnetic field $B$ changes more abruptly. Even at baryon number density $\rho=0.01 \mathrm{fm}^{-3}$ and $\alpha=0.01$ (black solid line), the strength of magnetic field is $1 \times 10^{14} \mathrm{G}$. This indicates that parameter $\gamma$ strongly affects the strength of the magnetic field near the surface of an NS. Furthermore, the radius at $1.4 M_{\odot}$ becomes larger when $\alpha$ becomes larger.

Next, the magnetic field $B(\rho)$ as a function of baryon number density $\rho$ for various $\gamma$ parameters is shown in Figure 3. Each line indicates a magnetic field with $B_{0}=2.5 \times 10^{18} \mathrm{G}$ and $\alpha=0.05$. For $\rho>0.3 \mathrm{fm}^{-3}, B(\rho)$ is almost constant $B_{0}=2.5 \times 10^{18} \mathrm{G}$. Here, magnetic field strengths at saturation density $\rho_{0}$ are given as $B\left(\rho_{0}\right)=1.23 \times 10^{17} \mathrm{G}$ for any value of $\gamma$. Magnetic field strengths $B\left(\rho_{0}\right)$ at saturation density $\rho_{0}$ for various $\alpha$ parameters are shown in Table 4.

The maximum mass, radius at $1.4 M_{\odot}$, and radius at $2.072 M_{\odot}$ by changing $\alpha$ and $\gamma$ in Equation (13) are shown in Tables 5-12 for each EoSs. The MR relations by changing $\alpha$ and $\gamma$ are given in Figures 4-10 for those EoSs with various incompressibilities, which satisfy the observational constraints. 


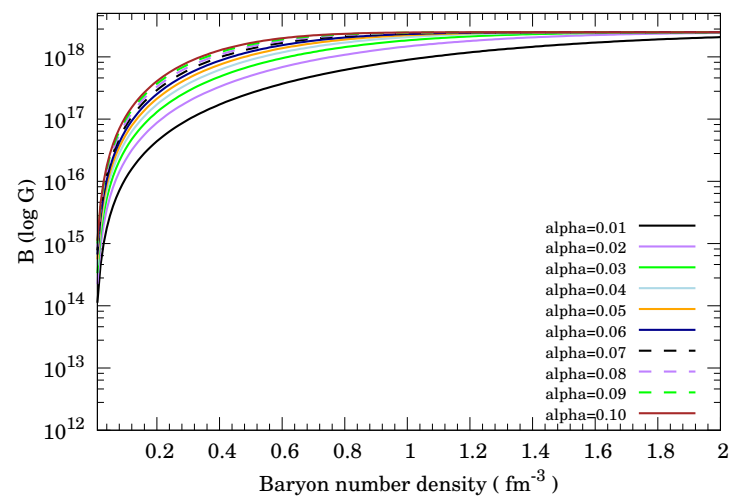

Figure 2. $\mathrm{B}(\rho)$ as a function of baryon number density $\rho$ for various $\alpha$ parameters with $B_{0}=2.5 \times 10^{18} \mathrm{G}$ and $\gamma=2$. The colored lines represent the following: black (solid line), magnetic fields $B(\rho)$ with $\alpha=0.01$; purple (solid line), magnetic fields $B(\rho)$ with $\alpha=0.02$; green (solid line), magnetic fields $B(\rho)$ with $\alpha=0.03$; light blue (solid line), magnetic fields $B(\rho)$ with $\alpha=0.04$; orange (solid line), magnetic fields $B(\rho)$ with $\alpha=0.05$; dark blue (solid line), magnetic fields $B(\rho)$ with $\alpha=0.06$; black (dashed line), magnetic fields $B(\rho)$ with $\alpha=0.07$; purple (dashed line), magnetic fields $B(\rho)$ with $\alpha=0.08$; green (dashed line), magnetic fields $B(\rho)$ with $\alpha=0.09$; brown (solid line), magnetic fields $B(\rho)$ with $\alpha=0.10$.

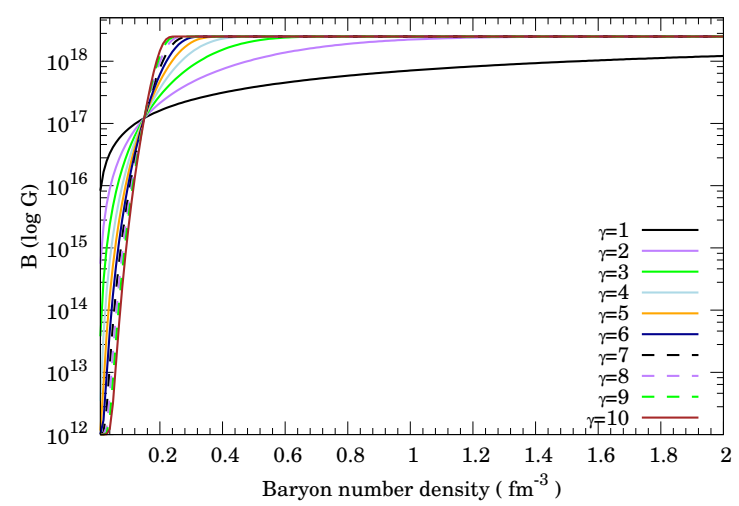

Figure 3. $\mathrm{B}(\rho)$ as a function of baryon number density $\rho$ for various $\gamma$ parameters with $B_{0}=2.5 \times 10^{18} \mathrm{G}$ and $\alpha=0.05$. The colored lines represent the following: black (solid line), magnetic fields $B(\rho)$ with $\gamma=1$; purple (solid line), magnetic fields $B(\rho)$ with $\gamma=2$; green (solid line), magnetic fields $B(\rho)$ with $\gamma=3$; light blue (solid line), magnetic fields $B(\rho)$ with $\gamma=4$; orange (solid line), magnetic fields $B(\rho)$ with $\gamma=5$; yellow (solid line), magnetic fields $B(\rho)$ with $\gamma=6$; dark blue (solid line), magnetic fields $B(\rho)$ with $\gamma=7$; red (solid line), magnetic fields $B(\rho)$ with $\gamma=8$; purple (dashed line), magnetic fields $B(\rho)$ with $\gamma=9$; brown (solid line), magnetic fields $B(\rho)$ with $\gamma=10$.

Table 4. Strengths of the magnetic field at saturation density $\rho_{0}=0.153 \mathrm{fm}^{-3}$ ( $B\left(\rho_{0}\right)$ in unit of Gauss) for 12 EoSs with $B_{0}=2.5 \times 10^{18} \mathrm{G}$.

\begin{tabular}{ll}
\hline$\alpha$ & $B\left(\rho_{0}\right)$ \\
\hline 0.01 & $2.59 \times 10^{16}$ \\
0.02 & $5.05 \times 10^{16}$ \\
0.03 & $7.79 \times 10^{16}$ \\
0.04 & $9.90 \times 10^{16}$ \\
0.05 & $1.23 \times 10^{17}$ \\
0.06 & $1.47 \times 10^{17}$ \\
0.07 & $1.70 \times 10^{17}$ \\
0.08 & $1.93 \times 10^{17}$ \\
0.09 & $2.16 \times 10^{17}$ \\
\hline
\end{tabular}


Figure 4 shows the MR relations of GM1 EoS with magnetic fields. The following EoSs go through $68 \%$ credibility with respect to the maximum mass and the radius at $1.4 M_{\odot}$.

1. For $\gamma=2, \alpha=0.05,0.06$, and 0.07 ;

2. For $\gamma=3, \alpha=0.02,0.03$, and 0.04 ;

3. For $\gamma=4, \alpha=0.01$.
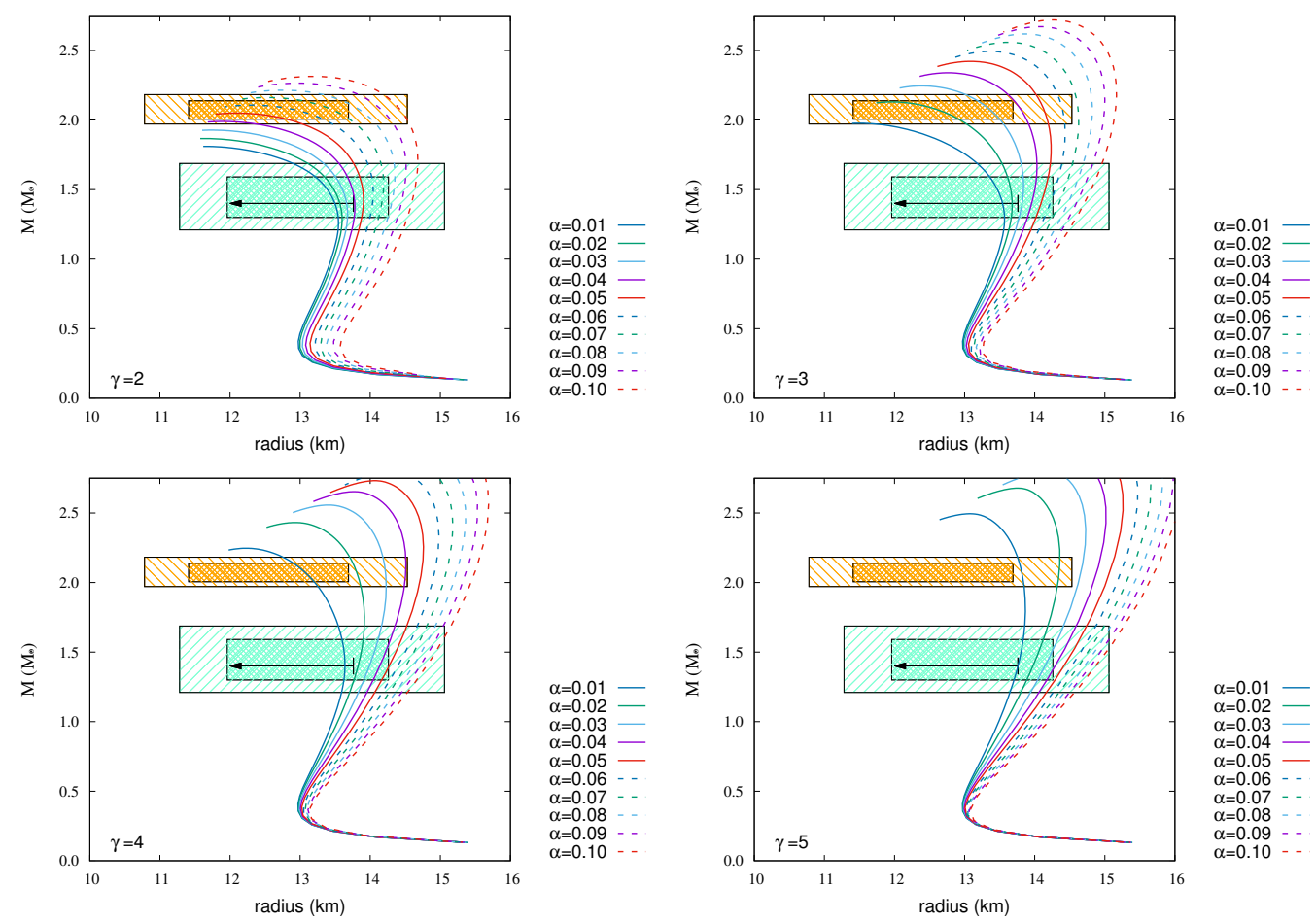

Figure 4. MR relations of GM1 EoS with magnetic fields. The strength of surface magnetic field strength $B_{s}$ is $10^{12} \mathrm{G}$, and the central magnetic field strength $B_{0}$ is $2.5 \times 10^{18} \mathrm{G}$. The arrow and colored hatched areas are the same as in Figure 1.

Figure 5 shows the MR relations of GM3 EoS with magnetic fields. The following EoSs go through $68 \%$ credibility with respect to the maximum mass and the radius at $1.4 M_{\odot}$.

1. For $\gamma=2, \alpha=0.09$;

2. For $\gamma=3, \alpha=0.03,0.04$, and 0.05;

3. For $\gamma=4, \alpha=0.01$ and 0.02 ;

4. For $\gamma=5, \alpha=0.01$.

Figure 6 shows the MR relations of NL3 $\omega \rho$-a EoS with magnetic fields. The following EoSs go through $68 \%$ credibility with respect to the maximum mass and the radius at $1.4 M_{\odot}$.

1. For $\gamma=1, \alpha=0.01$;

2. For $\gamma=2, \alpha=0.01$.

Figure 7 shows the MR relations of NL3 $\omega \rho-\mathrm{b}$ EoS with magnetic fields. In this case, no EoSs go through $68 \%$ credibility with respect to the maximum mass and the radius at $1.4 M_{\odot}$. 
Table 5. Maximum mass $\left(M_{\max }\right)$ in unit of $M_{\odot}$ and radius $(\mathrm{km})$ at $M=1.4 M_{\odot}\left(R_{1.4 M_{\odot}}\right)$ and radius $(\mathrm{km})$ at $M=2.072 M_{\odot}\left(R_{2.072} M_{\odot}\right)$ for GM1 EoS with magnetic fields $\left(B_{S}=10^{12} \mathrm{G}, B_{0}=2.5 \times 10^{18} \mathrm{G}\right)$.

\begin{tabular}{cccccccc}
\hline \multicolumn{2}{c}{$\gamma=2$} & \multicolumn{5}{c}{$\gamma=3$} \\
\hline $\boldsymbol{\alpha}$ & $\boldsymbol{M}_{\max }$ & $\boldsymbol{R}_{\mathbf{1 . 4} \boldsymbol{M}_{\odot}}$ & $\boldsymbol{R}_{\mathbf{2 . 0 7 2} \boldsymbol{M}_{\odot}}$ & $\boldsymbol{\alpha}$ & $\boldsymbol{M}_{\max }$ & $\boldsymbol{R}_{\mathbf{1 . 4} \boldsymbol{M}_{\odot}}$ & $\boldsymbol{R}_{\mathbf{2 . 0 7 2} \boldsymbol{M}_{\odot}}$ \\
\hline 0.01 & 1.810 & 13.50 & - & 0.01 & 1.977 & 13.54 & - \\
0.02 & 1.867 & 13.57 & - & 0.02 & 2.130 & 13.68 & 12.62 \\
0.03 & 1.927 & 13.66 & - & 0.03 & 2.245 & 13.82 & 13.37 \\
0.04 & 1.989 & 13.78 & - & 0.04 & 2.339 & 13.96 & 13.81 \\
0.05 & 2.049 & 13.90 & - & 0.05 & 2.421 & 14.09 & 14.13 \\
0.06 & 2.107 & 14.03 & 12.93 & 0.06 & 2.493 & 14.22 & 14.40 \\
0.07 & 2.161 & 14.16 & 13.47 & 0.07 & 2.558 & 14.35 & 14.62 \\
0.08 & 2.214 & 14.30 & 13.85 & 0.08 & 2.619 & 14.47 & 14.82 \\
0.09 & 2.265 & 14.44 & 14.16 & 0.09 & 2.671 & 14.59 & 15.00 \\
0.10 & 2.313 & 14.59 & 14.43 & 0.10 & 2.719 & 14.71 & 15.15 \\
\hline & & $\gamma=4$ & & & & $\gamma=5$ & \\
\hline $\boldsymbol{\alpha}$ & $\boldsymbol{M}_{\max }$ & $\boldsymbol{R}_{\mathbf{1 . 4} \boldsymbol{M}_{\odot}}$ & $\boldsymbol{R}_{\mathbf{2 . 0 7 2} \boldsymbol{M}_{\odot}}$ & $\boldsymbol{\alpha}$ & $\boldsymbol{M}_{\mathbf{m a x}}$ & $\boldsymbol{R}_{\mathbf{1 . 4}} \boldsymbol{M}_{\odot}$ & $\boldsymbol{R}_{\mathbf{2 . 0 7 2} \boldsymbol{M}_{\odot}}$ \\
\hline 0.01 & 2.247 & 13.64 & 13.07 & 0.01 & 2.494 & 13.76 & 13.82 \\
0.02 & 2.431 & 13.83 & 13.81 & 0.02 & 2.679 & 13.98 & 14.35 \\
0.03 & 2.557 & 13.99 & 14.22 & 0.03 & 2.799 & 14.15 & 14.66 \\
0.04 & 2.653 & 14.14 & 14.51 & 0.04 & 2.889 & 14.29 & 14.88 \\
0.05 & 2.732 & 14.27 & 14.73 & 0.05 & 2.960 & 14.42 & 15.07 \\
0.06 & 2.800 & 14.40 & 14.92 & 0.05 & 3.020 & 14.53 & 15.23 \\
0.07 & 2.862 & 14.51 & 15.08 & 0.07 & 3.072 & 14.63 & 15.37 \\
0.08 & 2.915 & 14.62 & 15.23 & 0.08 & 3.118 & 14.72 & 15.50 \\
0.09 & 2.962 & 14.71 & 15.37 & 0.09 & 3.160 & 14.80 & 15.61 \\
0.10 & 3.005 & 14.81 & 15.49 & 0.10 & 3.197 & 14.88 & 15.71 \\
\hline & & & & & & &
\end{tabular}
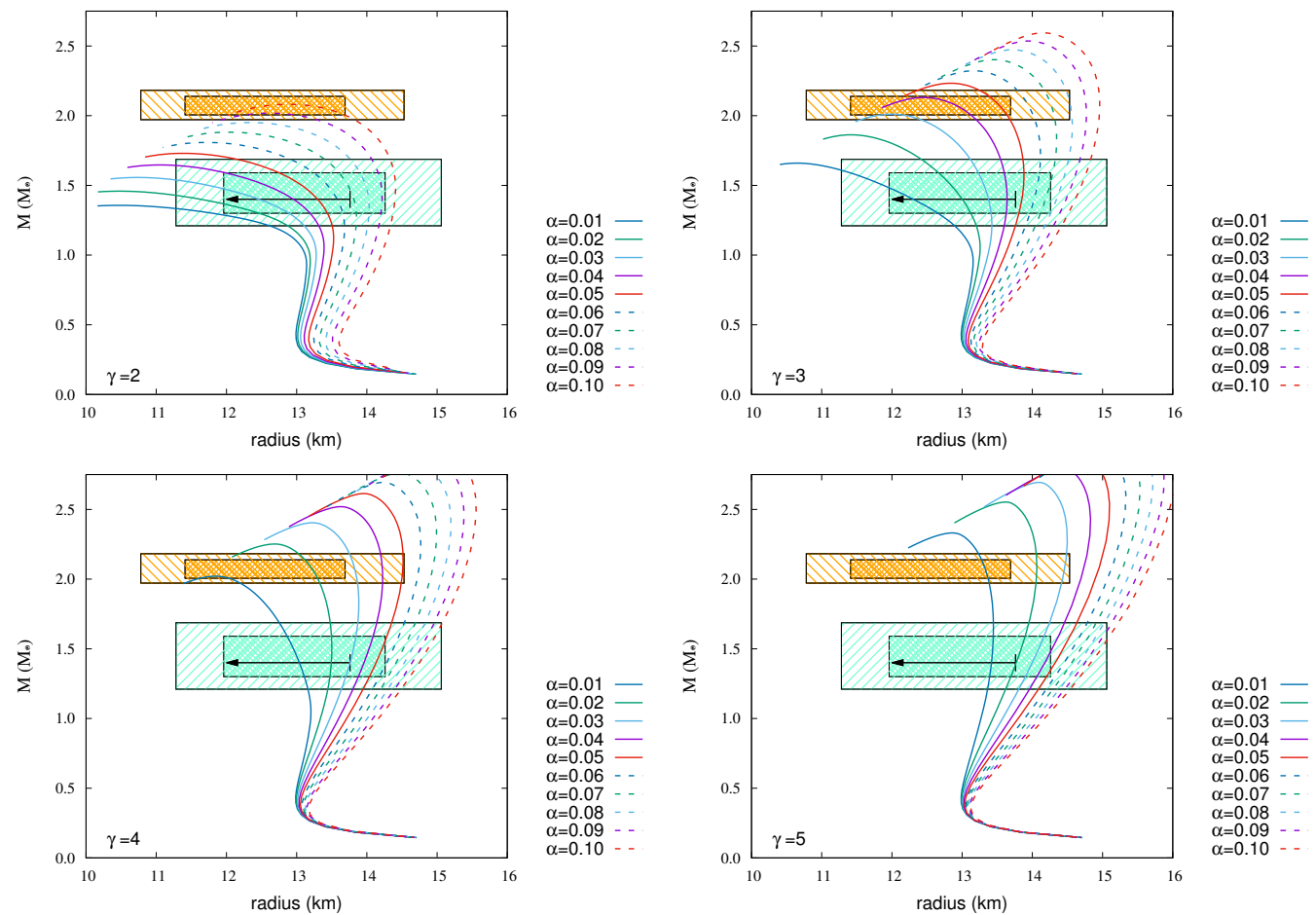

Figure 5. MR relations of GM3 EoS with magnetic fields. The strength of surface magnetic field strength $B_{s}$ is $10^{12} \mathrm{G}$, and the central magnetic field strength $B_{0}$ is $2.5 \times 10^{18} \mathrm{G}$. The arrow and colored hatched areas are the same as in Figure 1. 
Table 6. Maximum mass $\left(M_{\max }\right)$ in unit of $M_{\odot}$ and radius $(\mathrm{km})$ at $M=1.4 M_{\odot}\left(R_{1.4} M_{\odot}\right)$ and radius $(\mathrm{km})$ at $M=2.072 M_{\odot}\left(R_{2.072} M_{\odot}\right)$ for GM3 EoS with magnetic fields $\left(B_{S}=10^{12} \mathrm{G}, B_{0}=2.5 \times 10^{18} \mathrm{G}\right)$.

\begin{tabular}{cccccccc}
\hline \multicolumn{2}{c}{$\gamma=2$} & \multicolumn{5}{c}{$\gamma=3$} \\
\hline $\boldsymbol{\alpha}$ & $\boldsymbol{M}_{\max }$ & $\boldsymbol{R}_{\mathbf{1 . 4} \boldsymbol{M}_{\odot}}$ & $\boldsymbol{R}_{\mathbf{2 . 0 7 2} \boldsymbol{M}_{\odot}}$ & $\boldsymbol{\alpha}$ & $\boldsymbol{M}_{\max }$ & $\boldsymbol{R}_{\mathbf{1 . 4} \boldsymbol{M}_{\odot}}$ & $\boldsymbol{R}_{\mathbf{2 . 0 7 2} \boldsymbol{M}_{\odot}}$ \\
\hline 0.01 & 1.357 & - & - & 0.01 & 1.660 & 12.31 & - \\
0.02 & 1.458 & 11.63 & - & 0.02 & 1.863 & 13.00 & - \\
0.03 & 1.557 & 12.46 & - & 0.03 & 2.011 & 13.38 & - \\
0.04 & 1.647 & 12.95 & - & 0.04 & 2.130 & 13.64 & 12.92 \\
0.05 & 1.730 & 13.30 & - & 0.05 & 2.233 & 13.85 & 13.54 \\
0.06 & 1.808 & 13.58 & - & 0.06 & 2.322 & 14.03 & 13.95 \\
0.07 & 1.881 & 13.82 & - & 0.07 & 2.402 & 14.19 & 14.27 \\
0.08 & 1.951 & 14.03 & - & 0.08 & 2.473 & 14.34 & 14.54 \\
0.09 & 2.018 & 14.22 & - & 0.09 & 2.536 & 14.48 & 14.77 \\
0.10 & 2.081 & 14.40 & 13.19 & 0.10 & 2.594 & 14.61 & 14.96 \\
\hline & & $\gamma=4$ & & & & $\gamma=5$ & \\
\hline $\boldsymbol{\alpha}$ & $\boldsymbol{M}_{\max }$ & $\boldsymbol{R}_{\mathbf{1 . 4} \boldsymbol{M}_{\odot}}$ & $\boldsymbol{R}_{\mathbf{2 . 0 7 2} \boldsymbol{M}_{\odot}}$ & $\boldsymbol{\alpha}$ & $\boldsymbol{M}_{\mathbf{m a x}}$ & $\boldsymbol{R}_{\mathbf{1 . 4}} \boldsymbol{M}_{\odot}$ & $\boldsymbol{R}_{\mathbf{2 . 0 7 2} \boldsymbol{M}_{\odot}}$ \\
\hline 0.01 & 2.021 & 13.04 & & 0.01 & 2.768 & 13.84 & 14.36 \\
0.02 & 2.252 & 13.49 & 13.22 & 0.02 & 2.949 & 14.13 & 14.80 \\
0.03 & 2.404 & 13.76 & 13.83 & 0.03 & 3.060 & 14.31 & 15.07 \\
0.04 & 2.520 & 13.96 & 14.22 & 0.04 & 3.139 & 14.45 & 15.27 \\
0.05 & 2.614 & 14.13 & 14.51 & 0.05 & 3.201 & 14.57 & 15.42 \\
0.06 & 2.694 & 14.28 & 14.74 & 0.06 & 3.253 & 14.66 & 15.55 \\
0.07 & 2.763 & 14.42 & 14.94 & 0.07 & 3.297 & 14.75 & 15.66 \\
0.08 & 2.824 & 14.54 & 15.11 & 0.08 & 3.336 & 14.82 & 15.76 \\
0.09 & 2.878 & 14.65 & 15.26 & 0.09 & 3.370 & 14.89 & 15.85 \\
0.10 & 2.927 & 14.75 & 15.40 & 0.10 & 3.401 & 14.95 & 15.93 \\
\hline
\end{tabular}
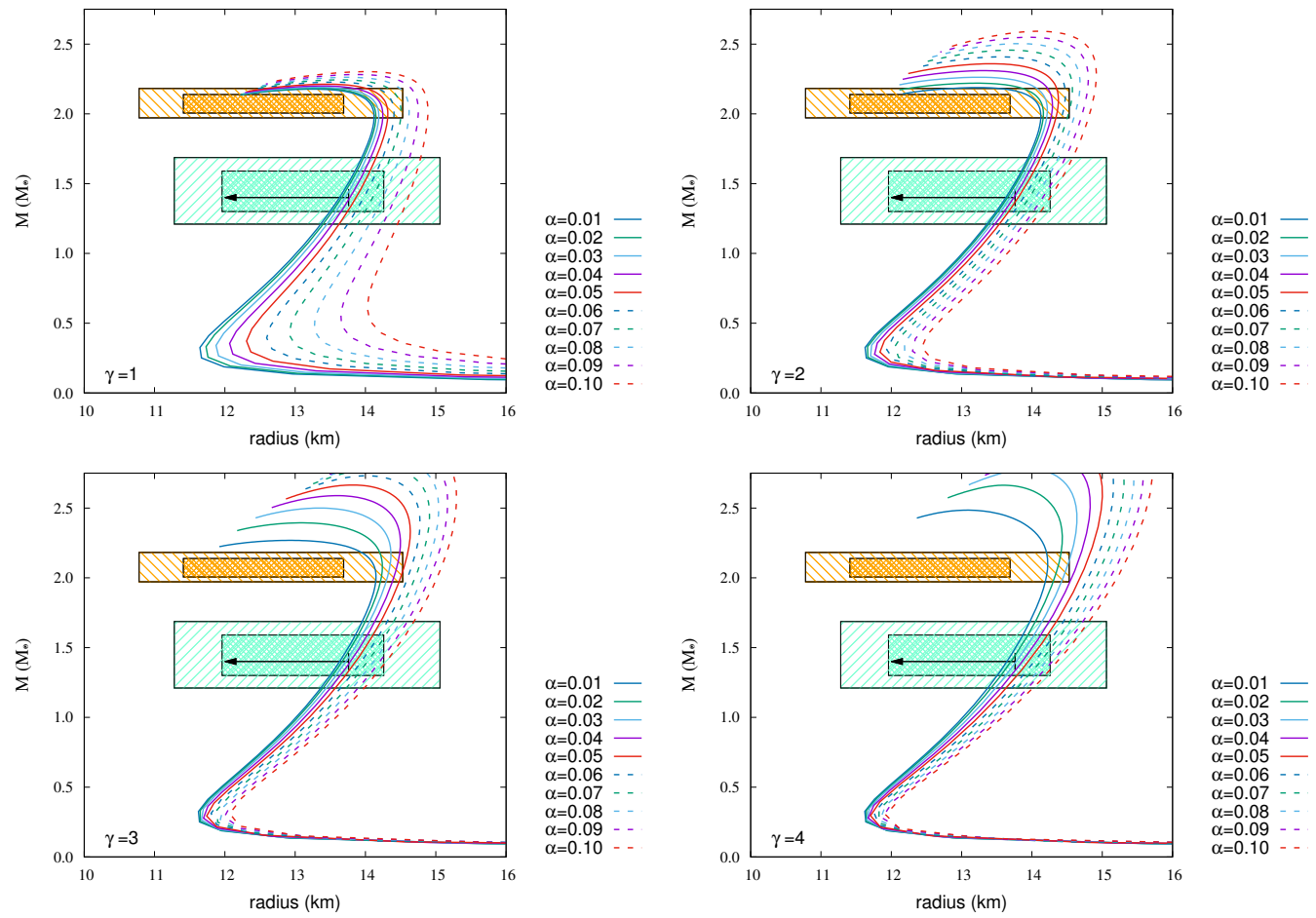

Figure 6. MR relations of NL3 $\omega \rho$-a EoS with magnetic fields. The strength of surface magnetic field strength $B_{s}$ is $10^{12} \mathrm{G}$, and the central magnetic field strength $B_{0}$ is $2.5 \times 10^{18} \mathrm{G}$. The arrow and colored hatched areas are the same as in Figure 1. 
Table 7. Maximum mass $\left(M_{\max }\right)$ in unit of $M_{\odot}$ and radius $(\mathrm{km})$ at $M=1.4 M_{\odot}\left(R_{1.4 M_{\odot}}\right)$ and radius $(\mathrm{km})$ at $M=2.072 M_{\odot}\left(R_{2.072} M_{\odot}\right)$ for NL3 $\omega \rho$-a EoS with magnetic fields $\left(B_{S}=10^{12} \mathrm{G}\right.$, $\left.B_{0}=2.5 \times 10^{18} \mathrm{G}\right)$.

\begin{tabular}{cccccccc}
\hline \multicolumn{2}{c}{$\gamma=1$} & \multicolumn{5}{c}{$\gamma=2$} \\
\hline $\boldsymbol{\alpha}$ & $\boldsymbol{M}_{\max }$ & $\boldsymbol{R}_{\mathbf{1 . 4} \boldsymbol{M}_{\odot}}$ & $\boldsymbol{R}_{\mathbf{2 . 0 7 2}} \boldsymbol{M}_{\odot}$ & $\boldsymbol{\alpha}$ & $\boldsymbol{M}_{\max }$ & $\boldsymbol{R}_{\mathbf{1 . 4} \boldsymbol{M}_{\odot}}$ & $\boldsymbol{R}_{\mathbf{2 . 0 7 2} \boldsymbol{M}_{\odot}}$ \\
\hline 0.01 & 2.177 & 13.63 & 14.07 & 0.01 & 2.188 & 13.63 & 14.08 \\
0.02 & 2.182 & 13.66 & 14.10 & 0.02 & 2.220 & 13.65 & 14.14 \\
0.03 & 2.190 & 13.71 & 14.15 & 0.03 & 2.262 & 13.69 & 14.21 \\
0.04 & 2.200 & 13.78 & 14.21 & 0.04 & 2.310 & 13.74 & 14.29 \\
0.05 & 2.212 & 13.87 & 14.29 & 0.05 & 2.359 & 13.81 & 14.38 \\
0.06 & 2.227 & 13.98 & 14.39 & 0.06 & 2.409 & 13.88 & 14.47 \\
0.07 & 2.244 & 14.10 & 14.49 & 0.07 & 2.457 & 13.96 & 14.55 \\
0.08 & 2.262 & 14.25 & 14.61 & 0.08 & 2.503 & 14.05 & 14.65 \\
0.09 & 2.282 & 14.42 & 14.75 & 0.09 & 2.549 & 14.16 & 14.75 \\
0.10 & 2.303 & 14.61 & 14.88 & 0.10 & 2.592 & 14.26 & 14.86 \\
\hline & & $\gamma=3$ & & & & $\gamma=4$ & \\
\hline $\boldsymbol{\alpha}$ & $\boldsymbol{M}_{\max }$ & $\boldsymbol{R}_{\mathbf{1 . 4}} \boldsymbol{M}_{\odot}$ & $\boldsymbol{R}_{\mathbf{2 . 0 7 2}} \boldsymbol{M}_{\odot}$ & $\boldsymbol{\alpha}$ & $\boldsymbol{M}_{\mathbf{m a x}}$ & $\boldsymbol{R}_{\mathbf{1 . 4}} \boldsymbol{M}_{\odot}$ & $\boldsymbol{R}_{\mathbf{2 . 0 7 2} \boldsymbol{M}_{\odot}}$ \\
\hline 0.01 & 2.268 & 13.63 & 14.13 & 0.01 & 2.485 & 13.64 & 14.22 \\
0.02 & 2.394 & 13.67 & 14.24 & 0.02 & 2.664 & 13.70 & 14.37 \\
0.03 & 2.500 & 13.72 & 14.34 & 0.03 & 2.782 & 13.78 & 14.50 \\
0.04 & 2.589 & 13.79 & 14.44 & 0.04 & 2.869 & 13.86 & 14.63 \\
0.05 & 2.665 & 13.86 & 14.55 & 0.05 & 2.939 & 13.95 & 14.75 \\
0.06 & 2.731 & 13.94 & 14.65 & 0.06 & 2.997 & 14.04 & 14.87 \\
0.07 & 2.789 & 14.03 & 14.76 & 0.07 & 3.047 & 14.12 & 14.98 \\
0.08 & 2.841 & 14.12 & 14.87 & 0.08 & 3.089 & 14.21 & 15.09 \\
0.09 & 2.887 & 14.21 & 14.97 & 0.09 & 3.127 & 14.29 & 15.19 \\
0.10 & 2.930 & 14.30 & 15.08 & 0.10 & 3.161 & 14.37 & 15.29 \\
\hline & & & & & & &
\end{tabular}
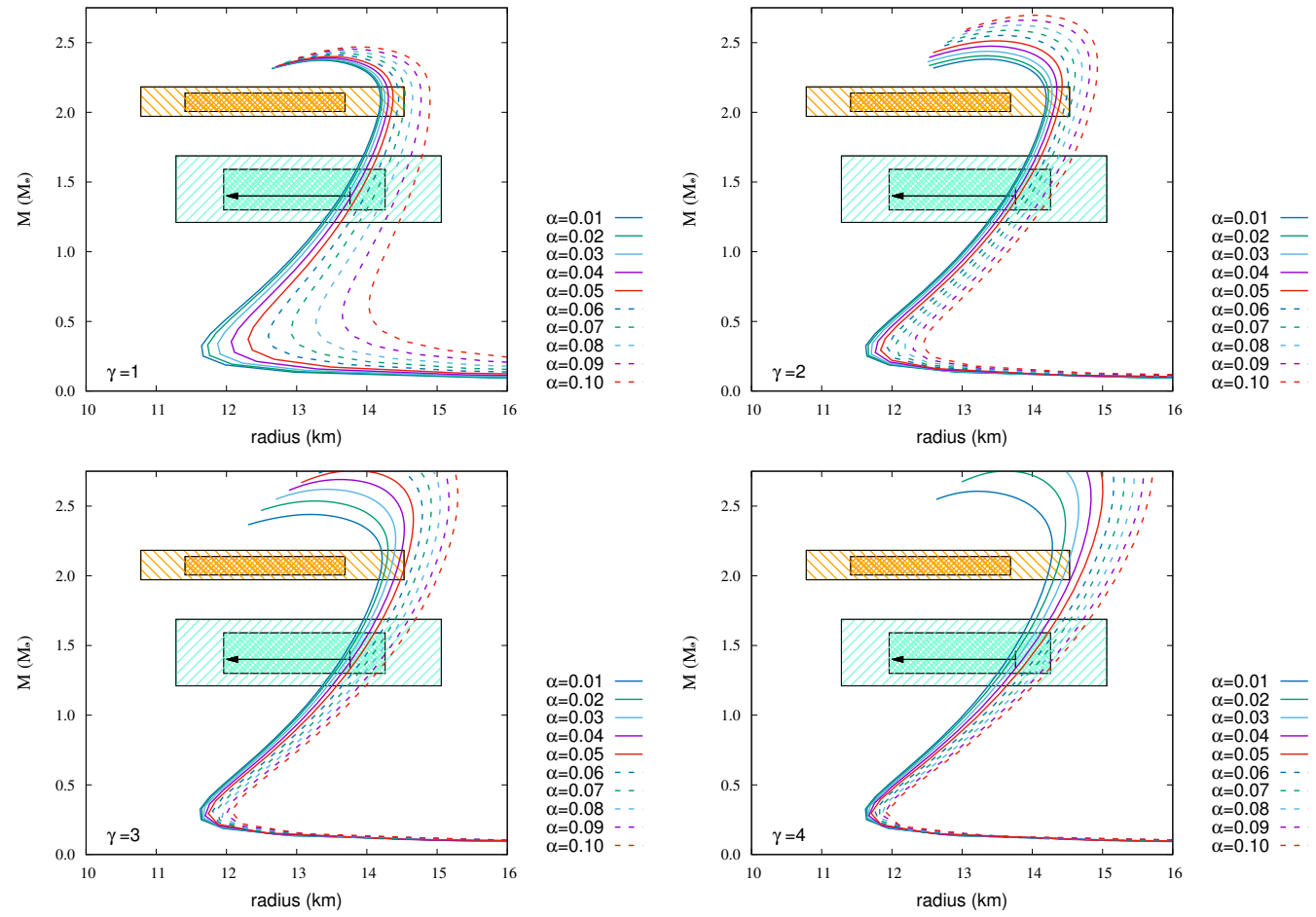

Figure 7. MR relations of NL3 $\omega \rho-\mathrm{b}$ EoS with magnetic fields. The strength of surface magnetic field strength $B_{S}$ is $10^{12} \mathrm{G}$, and the central magnetic field strength $B_{0}$ is $2.5 \times 10^{18} \mathrm{G}$. The arrow and colored hatched areas are the same as in Figure 1. 
Table 8. Maximum mass $\left(M_{\max }\right)$ in unit of $M_{\odot}$ and radius $(\mathrm{km})$ at $M=1.4 M_{\odot}\left(R_{1.4 M_{\odot}}\right)$ and radius $(\mathrm{km})$ at $M=2.072 M_{\odot}\left(R_{2.072} M_{\odot}\right)$ for NL3 $\omega \rho-\mathrm{b}$ EoS with magnetic fields $\left(B_{S}=10^{12} \mathrm{G}\right.$, $\left.B_{0}=2.5 \times 10^{18} \mathrm{G}\right)$.

\begin{tabular}{|c|c|c|c|c|c|c|c|}
\hline \multicolumn{4}{|c|}{$\gamma=1$} & \multicolumn{4}{|c|}{$\gamma=2$} \\
\hline$\alpha$ & $M_{\max }$ & $R_{1.4 M_{\odot}}$ & $R_{2.072 M_{\odot}}$ & $\alpha$ & $M_{\max }$ & $R_{1.4 M_{\odot}}$ & $R_{2.072} M_{\odot}$ \\
\hline 0.01 & 2.373 & 13.63 & 14.19 & 0.01 & 2.381 & 13.63 & 14.19 \\
\hline 0.02 & 2.377 & 13.66 & 14.21 & 0.02 & 2.405 & 13.65 & 14.23 \\
\hline 0.03 & 2.383 & 13.71 & 14.25 & 0.03 & 2.438 & 13.69 & 14.27 \\
\hline 0.04 & 2.390 & 13.78 & 14.30 & 0.04 & 2.475 & 13.74 & 14.33 \\
\hline 0.05 & 2.400 & 13.87 & 14.37 & 0.05 & 2.512 & 13.81 & 14.39 \\
\hline 0.06 & 2.411 & 13.98 & 14.45 & 0.06 & 2.551 & 13.88 & 14.47 \\
\hline 0.07 & 2.424 & 14.10 & 14.54 & 0.07 & 2.588 & 13.96 & 14.56 \\
\hline 0.08 & 2.438 & 14.25 & 14.65 & 0.08 & 2.625 & 14.05 & 14.65 \\
\hline 0.09 & 2.453 & 14.42 & 14.77 & 0.09 & 2.661 & 14.16 & 14.75 \\
\hline 0.10 & 2.469 & 14.61 & 14.90 & 0.10 & 2.696 & 14.26 & 14.86 \\
\hline \multicolumn{4}{|c|}{$\gamma=3$} & \multicolumn{4}{|c|}{$\gamma=4$} \\
\hline$\alpha$ & $M_{\max }$ & $R_{1.4 M_{\odot}}$ & $R_{2.072 M_{\odot}}$ & $\alpha$ & $M_{\max }$ & $R_{1.4 M_{\odot}}$ & $R_{2.072} M_{\odot}$ \\
\hline 0.01 & 2.440 & 13.63 & 14.21 & 0.01 & 2.606 & 13.64 & 14.25 \\
\hline 0.02 & 2.536 & 13.67 & 14.27 & 0.02 & 2.751 & 13.70 & 14.37 \\
\hline 0.03 & 2.620 & 13.72 & 14.35 & 0.03 & 2.848 & 13.78 & 14.50 \\
\hline 0.04 & 2.691 & 13.79 & 14.45 & 0.04 & 2.924 & 13.86 & 14.63 \\
\hline 0.05 & 2.754 & 13.86 & 14.55 & 0.05 & 2.984 & 13.95 & 14.75 \\
\hline 0.06 & 2.808 & 13.94 & 14.65 & 0.06 & 3.033 & 14.04 & 14.87 \\
\hline 0.07 & 2.855 & 14.03 & 14.76 & 0.07 & 3.076 & 14.12 & 14.98 \\
\hline 0.08 & 2.900 & 14.12 & 14.87 & 0.08 & 3.114 & 14.21 & 15.09 \\
\hline 0.09 & 2.940 & 14.21 & 14.97 & 0.09 & 3.149 & 14.29 & 15.19 \\
\hline 0.10 & 2.977 & 14.30 & 15.08 & 0.10 & 3.180 & 14.37 & 15.29 \\
\hline
\end{tabular}

Figure 8 shows the MR relations of TM2 $\omega \rho$-a EoS with magnetic fields. The following EoSs go through $68 \%$ credibility with respect to the maximum mass and the radius $1.4 \mathrm{M}_{\odot}$.

1. For $\gamma=2, \alpha=0.05$ and 0.06 ;

2. For $\gamma=3, \alpha=0.02,0.03$, and 0.04;

3. For $\gamma=4, \alpha=0.01$.
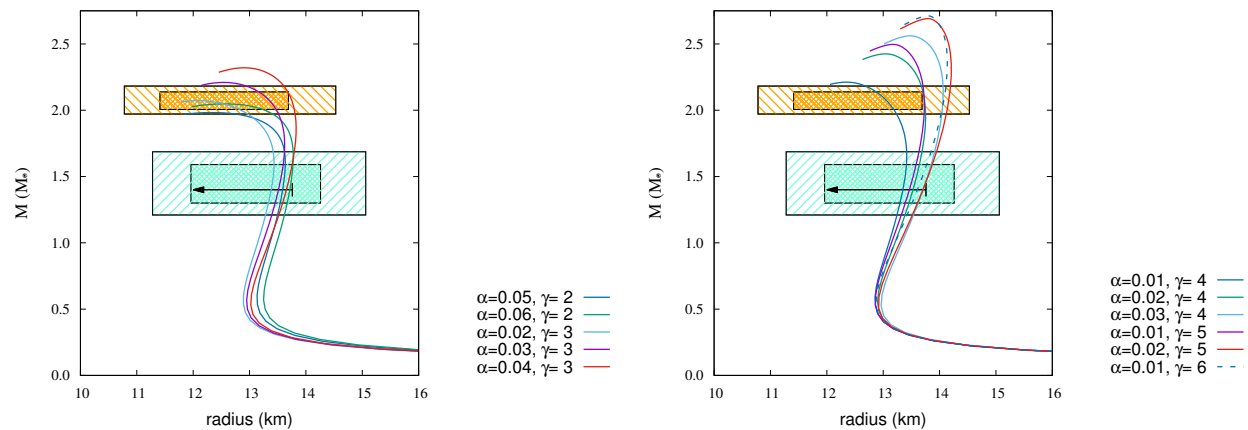

Figure 8. MR relations of TM2 $\omega \rho$-a EoS with magnetic fields. The strength of surface magnetic field strength $B_{S}$ is $10^{12} \mathrm{G}$, and the central magnetic field strength $B_{0}$ is $2.5 \times 10^{18} \mathrm{G}$. The arrow and colored hatched areas are the same as in Figure 1.

Figure 9 shows the MR relations of TM2 $\omega \rho-\mathrm{b}$ EoS with magnetic fields. The following EoSs go through $68 \%$ credibility with respect to the maximum mass and the radius at $1.4 M_{\odot}$.

1. For $\gamma=2, \alpha=0.03,0.04,0.05$, and 0.06 ;

2. For $\gamma=3, \alpha=0.01,0.02,0.03$, and 0.04;

3. For $\gamma=4, \alpha=0.01$. 
Table 9. Maximum mass $\left(M_{\max }\right)$ in unit of $M_{\odot}$ and radius $(\mathrm{km})$ at $M=1.4 M_{\odot}\left(R_{1.4 M_{\odot}}\right)$ and radius $(\mathrm{km})$ at $M=2.072 M_{\odot}\left(R_{2.072} M_{\odot}\right)$ for TM2 $\omega \rho$-a EoS with magnetic fields $\left(B_{S}=10^{12} \mathrm{G}\right.$, $\left.B_{0}=2.5 \times 10^{18} \mathrm{G}\right)$.

\begin{tabular}{ccccc}
\hline$\gamma$ & $\alpha$ & $\boldsymbol{M}_{\max }$ & $\boldsymbol{R}_{\mathbf{1 . 4}} \boldsymbol{M}_{\odot}$ & $\boldsymbol{R}_{\mathbf{2 . 0 7 2} \boldsymbol{M}_{\odot}}$ \\
\hline 2 & 0.05 & 1.983 & 13.59 & - \\
2 & 0.06 & 2.048 & 13.71 & 12.56 \\
\hline 3 & 0.02 & 2.072 & 13.38 & 12.12 \\
3 & 0.03 & 2.210 & 13.51 & 13.35 \\
3 & 0.04 & 2.320 & 13.65 & 13.75 \\
\hline 4 & 0.01 & 2.212 & 13.35 & 13.06 \\
4 & 0.02 & 2.425 & 13.53 & 13.73 \\
4 & 0.03 & 2.561 & 13.71 & 14.06 \\
\hline 5 & 0.01 & 2.497 & 13.46 & 13.73 \\
5 & 0.02 & 2.691 & 13.71 & 14.16 \\
\hline 6 & 0.01 & 2.711 & 13.61 & 14.08 \\
\hline
\end{tabular}
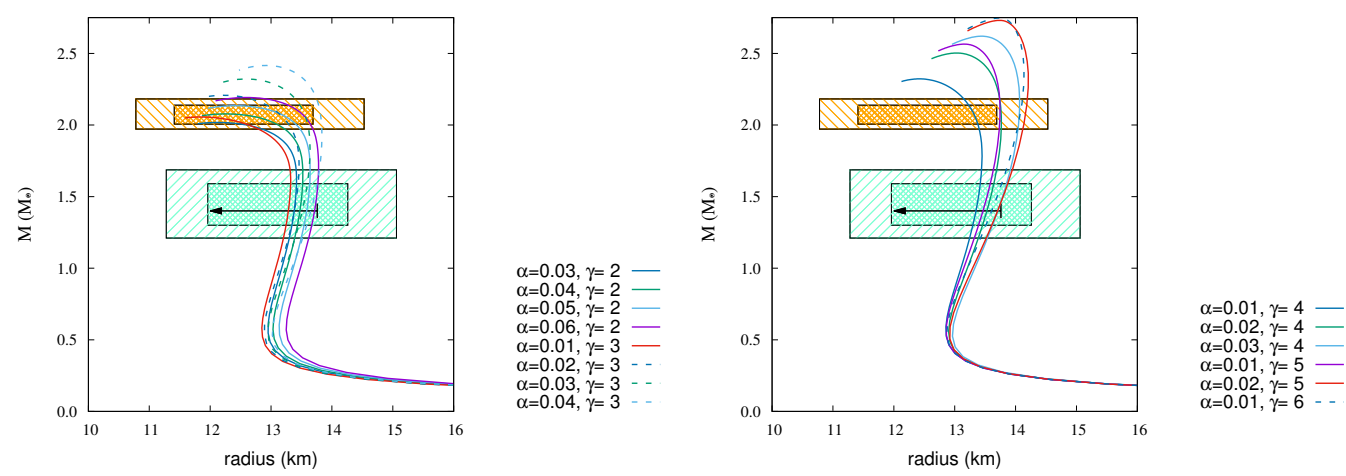

Figure 9. MR relations of TM $2 \omega \rho-\mathrm{b}$ EoS with magnetic fields. The strength of surface magnetic field strength $B_{S}$ is $10^{12} \mathrm{G}$, and the central magnetic field strength $B_{0}$ is $2.5 \times 10^{18} \mathrm{G}$. The arrow and colored hatched areas are the same as in Figure 1.

Table 10. Maximum mass $\left(M_{\max }\right)$ in unit of $M_{\odot}$ and radius $(\mathrm{km})$ at $M=1.4 M_{\odot}\left(R_{1.4 M_{\odot}}\right)$ and radius $(\mathrm{km})$ at $M=2.072 M_{\odot}\left(R_{2.072} M_{\odot}\right)$ for TM2 $\omega \rho$-b EoS with magnetic fields $\left(B_{S}=10^{12} \mathrm{G}\right.$, $\left.B_{0}=2.5 \times 10^{18} \mathrm{G}\right)$.

\begin{tabular}{ccccc}
\hline$\gamma$ & $\boldsymbol{\alpha}$ & $\boldsymbol{M}_{\max }$ & $\boldsymbol{R}_{\mathbf{1 . 4} \boldsymbol{M}_{\odot}}$ & $\boldsymbol{R}_{\mathbf{2 . 0 7 2} \boldsymbol{M}_{\odot}}$ \\
\hline 2 & 0.03 & 2.018 & 13.38 & - \\
2 & 0.04 & 2.077 & 13.47 & 12.51 \\
2 & 0.05 & 2.135 & 13.59 & 13.18 \\
2 & 0.06 & 2.192 & 13.71 & 13.52 \\
\hline 3 & 0.01 & 2.056 & 13.28 & - \\
3 & 0.02 & 2.207 & 13.38 & 13.15 \\
3 & 0.03 & 2.321 & 13.51 & 13.55 \\
3 & 0.04 & 2.416 & 13.65 & 13.81 \\
\hline 4 & 0.01 & 2.322 & 13.35 & 13.76 \\
4 & 0.02 & 2.503 & 13.53 & 14.06 \\
\hline 4 & 0.03 & 2.620 & 13.71 & 13.74 \\
\hline 5 & 0.01 & 2.565 & 13.46 & 14.16 \\
\hline 5 & 0.02 & 2.731 & 13.71 & 14.08 \\
\hline
\end{tabular}



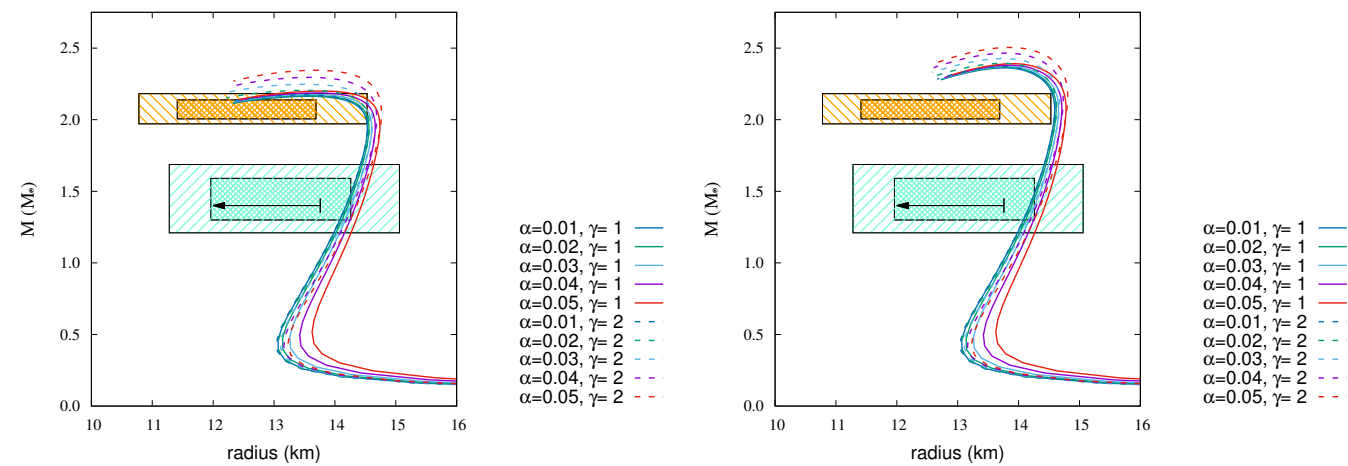

Figure 10. MR relations of DDME2-a (left) and DDME2-b (right) EoSs with magnetic fields The strength of surface magnetic field strength $B_{S}$ is $10^{12} \mathrm{G}$, and the central magnetic field strength $B_{0}$ is $2.5 \times 10^{18} \mathrm{G}$. The arrow and colored hatched areas are the same as in Figure 1 .

Table 11. Maximum mass $\left(M_{\max }\right)$ in unit of $M_{\odot}$ and radius $(\mathrm{km})$ at $M=1.4 M_{\odot}\left(R_{1.4 M_{\odot}}\right)$ and radius $(\mathrm{km})$ at $M=2.072 M_{\odot}\left(R_{2.072} M_{\odot}\right)$ for DDME2-a EoS with magnetic fields $\left(B_{S}=10^{12} \mathrm{G}_{,}\right.$ $\left.B_{0}=2.5 \times 10^{18} \mathrm{G}\right)$.

\begin{tabular}{cccccccc}
\hline \multicolumn{1}{c}{$\gamma=\mathbf{1}$} & \multicolumn{5}{c}{$\gamma=\mathbf{2}$} \\
\hline $\boldsymbol{\alpha}$ & $\boldsymbol{M}_{\max }$ & $\boldsymbol{R}_{\mathbf{1 . 4} \boldsymbol{M}_{\odot}}$ & $\boldsymbol{R}_{\mathbf{2 . 0 7 2} \boldsymbol{M}_{\odot}}$ & $\boldsymbol{\alpha}$ & $\boldsymbol{M}_{\max }$ & $\boldsymbol{R}_{\mathbf{1 . 4} \boldsymbol{M}_{\odot}}$ & $\boldsymbol{R}_{\mathbf{2 . 0 7 2} \boldsymbol{M}_{\odot}}$ \\
\hline 0.01 & 2.163 & 14.18 & 14.43 & 0.01 & 2.174 & 14.18 & 14.45 \\
0.02 & 2.168 & 14.22 & 14.47 & 0.02 & 2.205 & 14.21 & 14.51 \\
0.03 & 2.176 & 14.27 & 14.52 & 0.03 & 2.247 & 14.24 & 14.59 \\
0.04 & 2.187 & 14.34 & 14.59 & 0.04 & 2.295 & 14.30 & 14.68 \\
0.05 & 2.200 & 14.44 & 14.68 & 0.05 & 2.345 & 14.36 & 14.76 \\
\hline
\end{tabular}

Table 12. Maximum mass $\left(M_{\max }\right)$ in unit of $M_{\odot}$ and radius $(\mathrm{km})$ at $M=1.4 M_{\odot}\left(R_{1.4} M_{\odot}\right)$ and radius $(\mathrm{km})$ at $M=2.072 M_{\odot}\left(R_{2.072} M_{\odot}\right)$ for DDME2-b EoS with magnetic fields $\left(B_{S}=10^{12} \mathrm{G}\right.$, $\left.B_{0}=2.5 \times 10^{18} \mathrm{G}\right)$.

\begin{tabular}{cccccccc}
\hline \multicolumn{1}{c}{$\gamma=1$} & \multicolumn{5}{c}{$\gamma=2$} \\
\hline $\boldsymbol{\alpha}$ & $\boldsymbol{M}_{\max }$ & $\boldsymbol{R}_{\mathbf{1 . 4} \boldsymbol{M}_{\odot}}$ & $\boldsymbol{R}_{\mathbf{2 . 0 7 2}} \boldsymbol{M}_{\odot}$ & $\boldsymbol{\alpha}$ & $\boldsymbol{M}_{\max }$ & $\boldsymbol{R}_{\mathbf{1 . 4}} \boldsymbol{M}_{\odot}$ & $\boldsymbol{R}_{\mathbf{2 . 0 7 2}} \boldsymbol{M}_{\odot}$ \\
\hline 0.01 & 2.362 & 14.18 & 14.59 & 0.01 & 2.370 & 14.18 & 14.60 \\
0.02 & 2.366 & 14.22 & 14.62 & 0.02 & 2.394 & 14.21 & 14.62 \\
0.03 & 2.372 & 14.27 & 14.66 & 0.03 & 2.427 & 14.24 & 14.67 \\
0.04 & 2.381 & 14.34 & 14.71 & 0.04 & 2.465 & 14.30 & 14.73 \\
0.05 & 2.391 & 14.44 & 14.78 & 0.05 & 2.505 & 14.36 & 14.79 \\
\hline
\end{tabular}

Figure 10 shows the MR relations of DDME2-a (left) and DDME2-b (right) EoSs with magnetic fields. The following EoSs for DDME2-a go through $68 \%$ credibility with respect to the maximum mass and the radius at $1.4 M_{\odot}$.

1. For $\gamma=1, \alpha=0.01,0.02$, and 0.03 ;

2. For $\gamma=2, \alpha=0.01$.

\section{Summary}

In this work, the MR relations for magnetized neutron stars (NSs) have been investigated using twelve EoSs (seven kinds of EoSs). The NSs consist of nucleons and hyperons. They are implemented with magnetic fields, whose forms are changed by the shape parameters $\alpha$ and $\gamma$. The $\alpha$ and $\gamma$ are the damping factor and the exponent of the density dependence of a magnetic field, respectively.

First, we have found that, without any magnetic fields, only NL3 $\omega \rho$-a EoS satisfies the constraint for mass and radius from observations. Second, we have found that many varieties of EoSs with the implement of strong magnetic fields give maximum masses over $2 M_{\odot}$ and satisfy the constraints from the observations at the same time. 
To summarize, using different shapes of the magnetic field, in this work, we have demonstrated that many kinds of EoSs with strong magnetic fields can satisfy the constraints on the maximum mass and the radius within the range by observations. Other EoSs with lower incompressibility $(K<240 \mathrm{MeV})$ than those EoSs examined in this work will be studied in future work.

Author Contributions: data curation, C.W.; Visualization, C.W.; Methodology C.W., N.Y. and S.E.; writing original draft preparation, C.W.; writing review and editing, C.W., N.Y. and S.E. All authors have read and agreed to the published version of the manuscript.

Funding: This research received no external funding.

Institutional Review Board Statement: Not applicable.

Informed Consent Statement: Not applicable.

Data Availability Statement: Not applicable.

Acknowledgments: This work was supported by a Grant-in-Aid for Scientific Research (C) (Grants No. 20K03925 and 20K03943) from the Japan Society for the Promotion of Science (JSPS), and also supported by Leading Initiative for Excellent Young Researchers, MEXT, Japan.

Conflicts of Interest: The author declares no conflict of interest.

\section{References}

1. Oppenheimer, J.R.; Volkoff, G.M. On Massive Neutron Cores. Phys. Rev. 1939, 55, 374. [CrossRef]

2. Tolman, R.C. Static Solutions of Einstein's Field Equations for Spheres of Fluid. Phys. Rev. 1939, 55, 364. [CrossRef]

3. Bethe, H.A.; Brown, G.E. Observational Constraints on the Maximum Neutron Star Mass. Astrophys. J. 1995, 445, L129. [CrossRef]

4. Muto, T.; Maruyama, T.; Tatsumi, T. Effects of three-baryon forces on kaon condensation in hyperon-mixed matter. Phys. Lett. B 2021, 820, 136587. [CrossRef]

5. $\quad$ Demorest, P.B.; Pennucci, T.; Ransom, S.M.; Roberts, M.S.E.; Hessels, J.W.T. A two-solar-mass neutron star measured using Shapiro delay. Nature 2010, 467, 1081-1083. [CrossRef] [PubMed]

6. Antoniadis, J.; Freire, P.C.C.; Wex, N.; Tauris, T.M.; Lynch, R.S.; van Kerkwijk, M.H.; Kramer, M.; Bassa, C.; Dhillon, V.S.; Driebe, T.; et al. A massive pulsar in a compact relativistic binary. Science 2013, 340, 1233232. [CrossRef] [PubMed]

7. Cromartie, H.T.; Fonseca, E.; Ransom, S.M.; Demorest, P.B.; Arzoumanian, Z.; Blumer, H.; Brook, P.R.; DeCesar, M.E.; Dolch, T.; Ellis, J.A.; et al. Relativistic Shapiro delay measurements of an extremely massive millisecond pulsar. Nat. Astron. 2020, 4, 72-76. [CrossRef]

8. Fonseca, E.; Cromartie, H.T.; Pennucci, T.T.; Ray, P.S.; Kirichenko, A.Y.; Ransom, S.M.; Demorest, P.B.; Stairs, I.H.; Arzoumanian, Z.; Guillemot, L.; et al. Refined Mass and Geometric Measurements of the High-Mass PSR J0740+6620. arXiv 2021, arXiv:2104.00880v2.

9. Riley, T.E.; Watts, A.L.; Ray, P.S.; Bogdanov, S.; Guillot, S.; Morsink, S.M.; Bilous, A.V.; Arzoumanian, Z.; Choudhury, D.; Deneva, J.S.; et al. A NICER View of the Massive Pulsar PSR J0740+6620 Informed by Radio Timing and XMM-Newton Spectroscopy. Astrop. J. Lett. 2021, 918, L27. [CrossRef]

10. Miller, M.C.; Lamb, F.K.; Dittmann, A.J.; Bogdanov, S.; Arzoumanian, Z.; Gendreau, K.C.; Guillot, S.; Ho, W.C.G.; Lattimer, J.M.; Loewenstein, M.; et al. The radius of psr J0740+6620 from nicer and XMM-Newton data. arXiv 2021, arXiv:2105.06979v1.

11. Yanase, K.; Yoshinaga, N.; Nakano, E.; Watanabe, C. Deformation of neutron stars due to poloidal magnetic fields. Prog. Theor. Exp. Phys. 2019, 8, 083E01. [CrossRef]

12. Watanabe, C.; Yanase, K.; Yoshinaga, N. Searching optimum equations of state of neutron star matter in strong magnetic fields with rotation. Prog. Theor. Exp. Phys. 2020, 10, 103E04. [CrossRef]

13. Olausen, S.A.; Kaspi, V.M. The McGill Magnetar Catalog. Astrophys. J. Suppl. 2014, 212, 22. [CrossRef]

14. McGill Online Magnetar Catalog. Available online: http://www.physics.mcgill.ca/pulsar/magnetar/main.html (accessed on 8 May 2012).

15. Annala, E.; Gorda, T.; Kurkela, A.; Vuorinen, A. Gravitational-Wave Constraints on the Neutron-Star-Matter Equation of State. Phys. Rev. Lett. 2018, 120, 172703. [CrossRef]

16. Fattoyev, F.J.; Piekarewicz, J.; Horowitz, C. Neutron Skins and Neutron Stars in the Multimessenger Era. Phys. Rev. Lett. 2018, 120, 172702. [CrossRef]

17. Bauswein, A.; Just, O.; Janka, H.-T.; Stergioulas, N. Neutron-star radius constraints from GW170817 and future detections. Astrop. Jour. Lett. 2017, 850, L34. [CrossRef]

18. Miller, M.C.; Lamb, F.K.; Dittmann, A.J.; Bogdanov, S.; Arzoumanian, Z.; Gendreau, K.C.; Guillot, S.; Harding, A.K.; Ho, W.C.G.; Lattimer, J.M.; et al. PSR J0030+0451 Mass and Radius from NICER Data and Implications for the Properties of Neutron Star Matter. Astrop. J. Lett. 2019, 887, L24. [CrossRef]

19. Glendenning, N.K. Compact Stars; Springer Science \& Business Media: Berlin/Heidelberg, Germany, 2012. 
20. Fortin, M.; Avancini, S.S.; Providência, C.; Vidaña, I. Hypernuclei and massive neutron stars. Phys. Rev. C 2017, 95, 065803. [CrossRef]

21. Glendenning, N.K. Vacuum polarization effects on nuclear matter and neutron stars. Nucl. Phys. A 1989, 493, 521-548. [CrossRef]

22. Glendenning, N.K.; Moszkowski, S.A. Reconciliation of neutron-star masses and binding of the $\Lambda$ in hypernuclei. Phys. Rev. Lett. 1991, 67, 2414. [CrossRef]

23. Sugahara, Y.; Toki, H. Relativistic mean-field theory for unstable nuclei with non-linear $\sigma$ and $\omega$ terms. Nucl. Phys. A 1994, 579, 557-572. [CrossRef]

24. Broderick, A.; Prakash, M.; Lattimer, J.M. The Equation of State of Neutron Star Matter in Strong Magnetic Fields. Astrophys. J. 2000, 537, 351. [CrossRef]

25. Rabhi, A.; Perez-Garcia, M.A.; Providência, C.; Vidaña, I. Magnetic susceptibility and magnetization properties of asymmetric nuclear matter in a strong magnetic field. Phys. Rev. C 2015, 91, 045803. [CrossRef]

26. Casali, R.H.; Castro, L.B.; Menezes, D.P. Hadronic and hybrid stars subject to density-dependent magnetic fields. Phys. Rev. C 2014, 89, 015805. [CrossRef]

27. Particle Data Group. Available online: http://pdg.lbl.gov/ (accessed on 8 May 2012).

28. Ducoin, C.; Margueron, J.; Providência, C.; Vidaẽa, I. Core-crust transition in neutron stars: Predictivity of density developments. Phys. Rev. C 2011, 83, 045810. [CrossRef]

29. Baldo, M.; Burgio, G.F. The nuclear symmetry energy. Prog. Part. Nucl. Phys. 2016, 91, 203. [CrossRef]

30. Bandyopadhyay, D.; Chakrabarty, S.; Pal, S. Quantizing Magnetic Field and Quark-Hadron Phase Transition in a Neutron Star. Phys. Rev. Lett. 1997, 79, 2176. [CrossRef]

31. Menezes, D.P.; Alloy, M.D. Maxwell equation violation by density dependent magnetic fields in neutron stars. arXiv 2016, arXiv:1607.07687. 